\section{Generalized Isocline Method of Plotting Phase-Plane Trajectories}

\section{B. L. DEEKSHATULU AND I. S. N. MLRTHY}

Abstract-By considering simultaneously the $N-x$ (or the $N-\dot{x}$ ), where $N=d \dot{x} / d x$ and the $\dot{x}-x$ planes, second order non-linear autonomous systems (not easily amendable to the existing methods) described by the differential equation $\ddot{x}=F(x, \dot{x})$ can be studied. Changes in system behavior due to changes in initial conditions, nonlinear dampling and restoring forces can easily be studied. Simple and general as it is, the method presented here is believed to be novel.

\section{INIRODLCTION}

A general second-order nonlinear autonomous system is governed by the differential equation

$$
\ddot{x}=F(x, \dot{x})
$$

If we denote the slope of a trajectory on the phase plane by $N(=d \dot{x} / d x),(1)$ reduces to

$$
\dot{y} \dot{x}=F(x, \dot{x})
$$

There are numerous methods to construct individual phase-plane trajectories starting from a given set of initial conditions (like the methods of Lienard [2], [3], Ku [4], and Pell [1]. But there are only a few techniques which permit the study of the behavior of certain class of nonlinear systems corresponding to many initial conditions. For example Szegö [5] suggested a procedure, which needs the plotting of curves of certain equations on a transparent paper which are used later for plotting the trajectories. Brodestly's [6] method involves the division of the phase plane into regions with positive and negative $d \dot{x} / d x$ and $d^{2} \dot{x} / d x^{2}$. Deekshatulu [7] made use of simple transformations to effect rapid plotting of trajectories on the entire phase plane or in any desired region. The existing isocline method necessitates plotting the curves of $\dot{x}$ vs. $x$ from (2) for different constant values of $N$, from which the phase trajectory may be constructed from any chosen initial condition, but plotting of $\dot{x}-x$ curves for any given value of $N$ is not always possible; for example, equations like $\ddot{x}+\cot \dot{x} x+\sin x=0$, and $\ddot{x}+\dot{x}^{3}+x^{3}+\log x=0$ do not yield to the existing isocline method. In what follows, a new, general and simple method of plotting phase-plane trajectories for secondorder nonlinear systems governed by (1) is presented.

\section{The Proposed Method}

Equation (2) is rewritten as follows:

$$
N=F(x, \dot{x}) / \dot{x}
$$

Then,

1) On the $N-x(N-\dot{x})$ plane, plot curves of $N$ vs $x(N$ vs. $\dot{x})$ for different constant values of $\dot{x}(x)$. This is always possible for any $F(x, \dot{x})$

2) Draw lines of constant $N$ say corresponding to $N=N_{1}, N_{2}$, $N_{3}, \cdots$. This amounts to drawing a number of horizontal lines parallel to the $x(\dot{x})$ axis.

3) Keeping any horizontal line in view, say corresponding to $N=N_{1}$, determine all possible points of intersection of this line with the curves of constant $\dot{x}(x)$ as obtained under 1 ).

4) Plot these points on the $\dot{x}-x$ plane and join through a smooth curve. This curve then represents the isocline curve for $N=N_{1}$. Similarly other isocline curves corresponding to $N=N_{2}, N_{3}, \cdots$ are obtained.

5) Small slope lines with slopes $N_{1}, N_{2}, N_{3}, \cdots$ are then marked on each of the respective isocline curves corresponding to $N=N_{1}, N_{2}$, $\nu_{3}, \ldots$. From these slope lines, the phase trajectory may be constructed for any given initial conditions or the entire phase portrait may be obtained.

To illustrate the procedure the two following simple examples are given:

Manuscript received June 29, 1964: revised September 23, 1964.

The authors are with the Dept. of Electrical Engineering. Indian Institute of Science, Bangalore. India.

\section{Example I}

Consider the system with nonlinear damping given by

$$
\ddot{x}+\dot{x}^{2}+x=0
$$

On the phase plane by the existing isocline method one should solve the quadratic equation in $\dot{x}$ namely $\dot{x}^{2}+N \dot{x}+x=0$ and both the roots are valid. By the present method the procedure is as follows:

1) On the $N-x$ plane, curves of $N$ vs. $x$ are plotted from the equation

$$
N=-\dot{x}-\frac{x}{\dot{x}}
$$

for particular values of $\dot{x}$, say $\dot{x}=0, \pm 1, \pm 2, \cdots$. This is a simple job since it amounts to drawing straight lines with different slopes and different shifts from the origin. See Fig. 1.

2) A number of horizontal lines corresponding to $N= \pm 1, \pm 2$, .. are then drawn. These are already present on the graph sheet. See Fig. 1.

3) Consider a horizontal straight line say for $N=1.5$. Determine all possible points of intersections ( $x$ and $x$ ) of this straight line with the curves obtained under 1$)$. These are $(0.5,-0.5),(0.5,-1.0)$, $(-1.0,0.5)(-1.0,-2.0),(-2.5,1.0),(-2.5,-2.5)$ and $(-7.0,2.0)$.

4) These points are plotted on the $\dot{x}-x$ plane, joined and the slope lines marked with slope $N=1.5$. The procedure is repeated for other values of $N$, and the corresponding isoclines (with the respective slope lines marked on them) are obtained on the phase plane. Joining these slope lines give the entire phase portrait as shown in Fig. 2. The system represents that if the initial point is interior to the separatrix, the motion is an oscillation (not a harmonic motion). The singular point is a center.

\section{Example II}

Consider the equation

$$
\ddot{x}+x \dot{x}+x=0
$$

we have $N=-x(\dot{x}+1) /$

In this case the curves of $N$ vis, $x$ for different values of $\dot{x}$ are straight lines through the origin. See Fig. 3. Therefore, the present technique is much simpler than the usual isocline method (which is applicable for this example) since latter method necessitates plotting of the curves of $\dot{x}$ vs. $x$ from the equation $\dot{x}=-x / N+x$ for different values of $N$. The complete trajectories are shown in Fig. 4.

\section{Discussion}

The following important points may be noted:

1) a) While the present method is generally applicable to any second-order nonlinear autonomous system, it is particularly useful for the following equations:

$$
\begin{aligned}
& \ddot{x}+f(\dot{x})+x=0 \\
& \ddot{x}+f(\dot{x}) x+x=0
\end{aligned}
$$

b) For equations of the type $\ddot{x}+f(\dot{x})+g(x)=0$ one would plot the $N$ is $x$ curves from the equation $N=-f(\dot{x})+g(x) / \dot{x}$ for different constant values of $\dot{x}$. Instead, a simple procedure would be to imagine a scale change of the $Y$ axis from $N$ to $N \dot{x}$ ( $\dot{x}$ being a constant each time) and to shift the curve of $g(x)$ with the proper shift $f(\dot{x})$ from the origin depending on the value of $\dot{x}$. The scale change of $N$ axis for each $\dot{x}$ is to be taken care of at the time of reading the points of intersections of the $N-x$ curves and the constant $N$ lines.

2) Effect of variation of nonlinear damping and/or nonlinear restoring forces on system performance can be studied easily by the proposed method since it amounts to only interpretation of the shifts (from the origin) or of the scale change on the $N$ axis. For example the $y-x$ curve for the two equations $\ddot{x}+h(\dot{x})+x=0$ and $\ddot{x}+f(\dot{x})+x=0$ are the same when the shifts are suitably interpreted.

3) The nonlinear functions can be given either analytically or in the form of curves.

4) Sometimes a $N-x$ curve and the constant $N$ line may run so close to one another (for a certain range of $x$ ) as to make their points of intersection difficult to locate. This is not a disadvantage but merely 


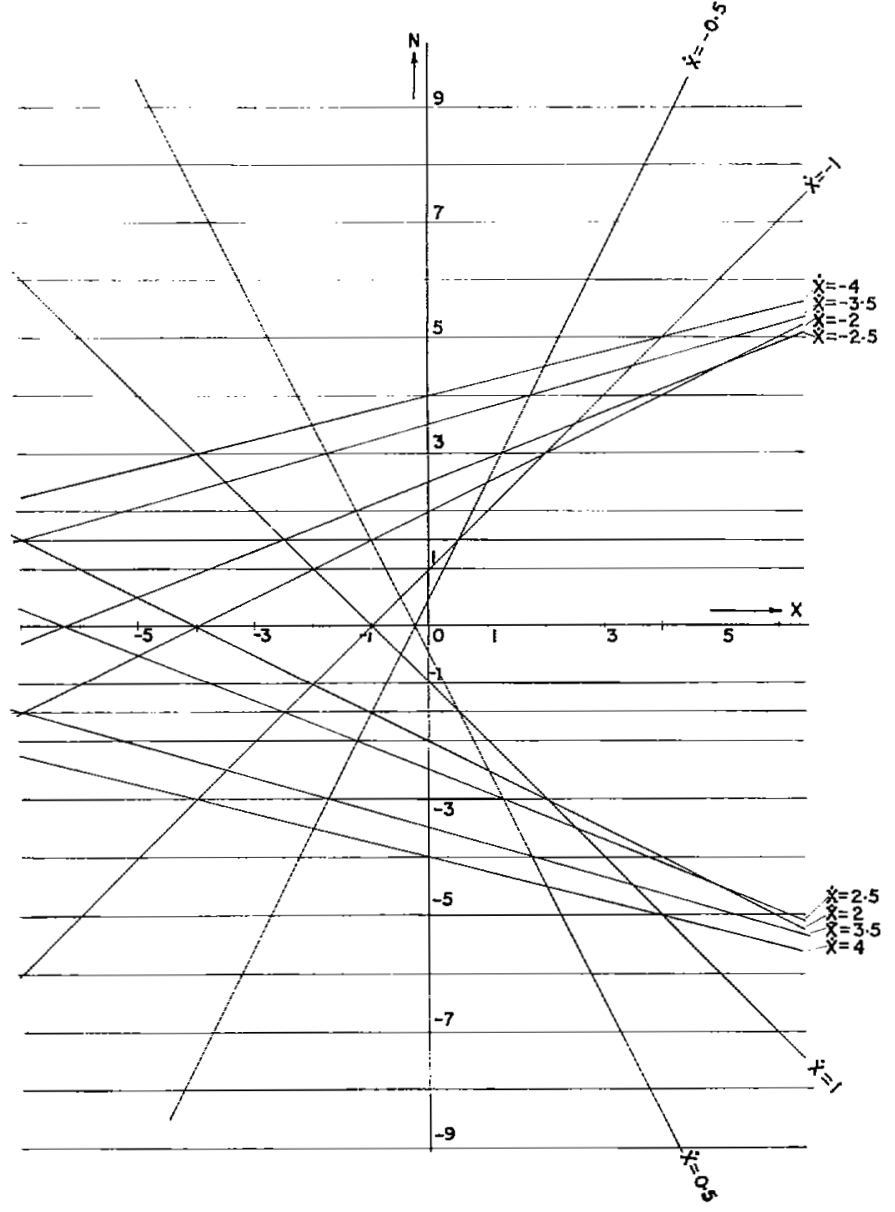

Fig. $1, x-x$ curves for constant $\dot{x}$ for the equation $\ddot{x}+\dot{x}^{2}+x=0$.

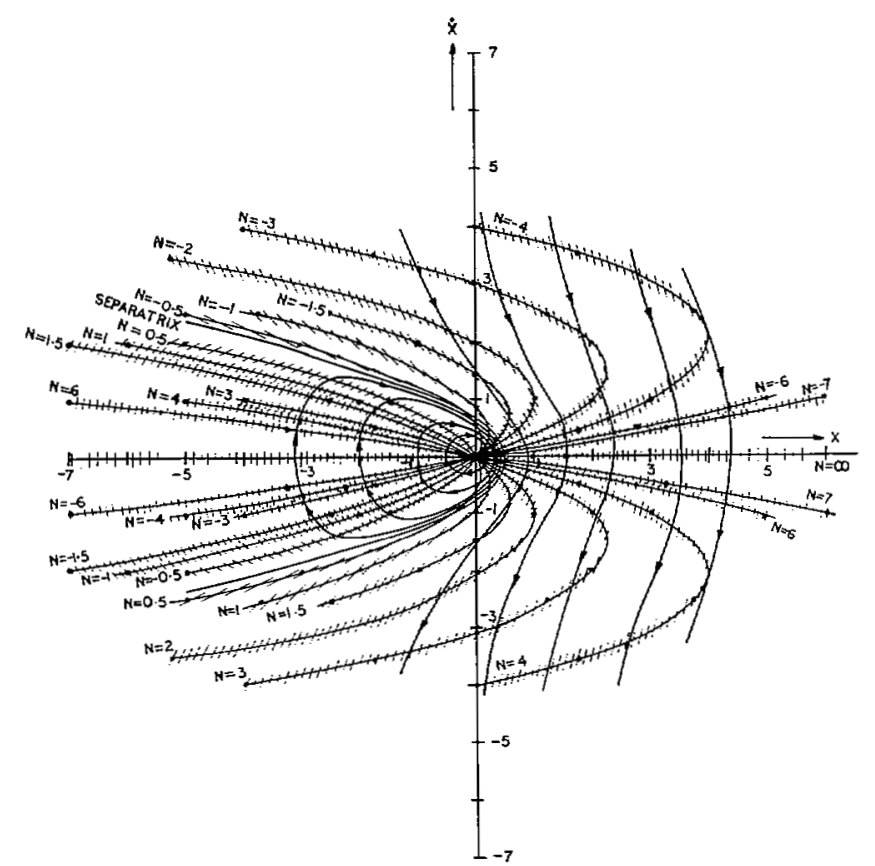

Fig. 2. Isoclines and phase-plane trajectories for the equation $\ddot{x}+\dot{x}^{2}+x=0$.

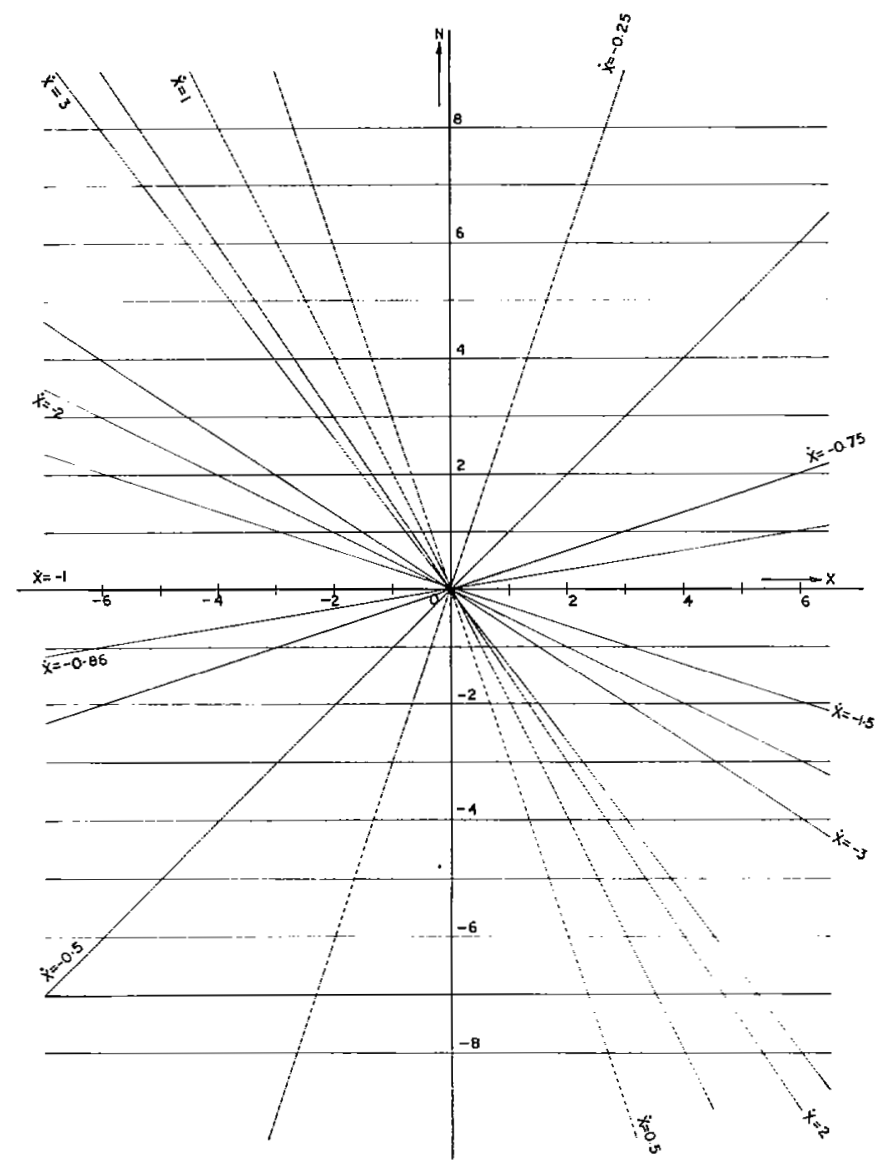

Fig. 3. $N-x$ curves for constant $\dot{x}$ for the equation $\ddot{x}+x \dot{x}+x=0$.

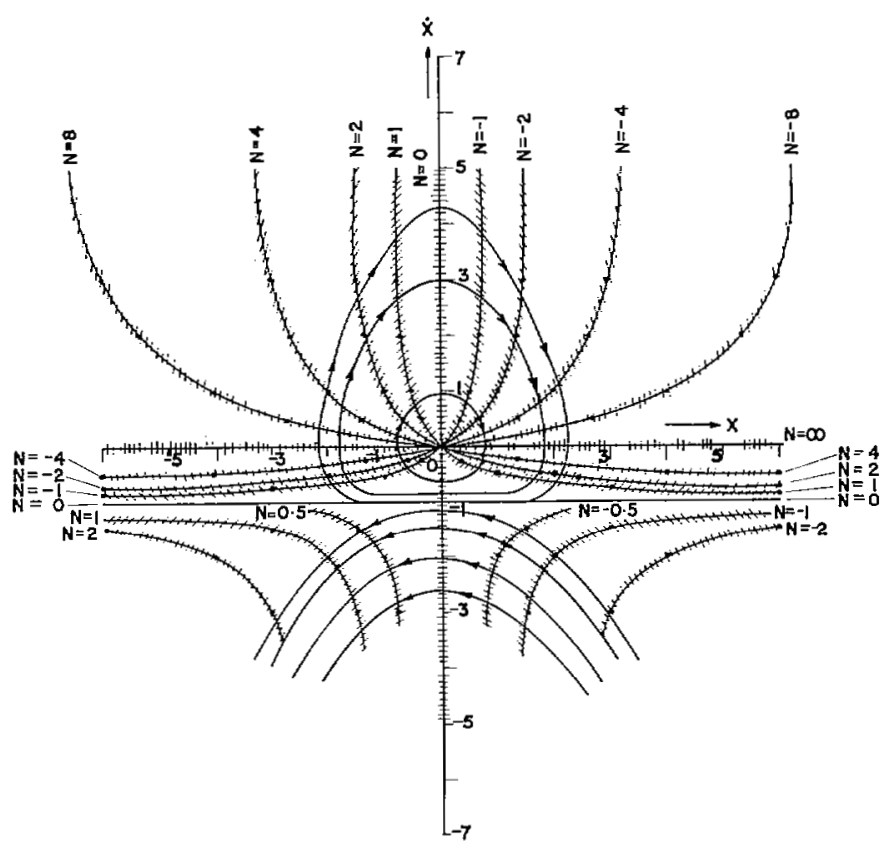

Fig. 4. Isoclines and phase-plane trajectories for the equation $\ddot{x}+x \dot{x}+x=0$. 
indicates that on the phase plane that isocline curve is nearly vertical in that range of $x$.

5) The well-known Leinard construction for plotting phase plane trajectories is clearly explained in 11 est [2] and Thaler and Pastel [3]. The Leinard procedure is a step by step construction for drawing any particular trajectory corresponding to a given set of initial conditions, whereas the present method determines the entire phase portrait indicating the system behavior for all initial conditions. Further, the Leinard construction (even for drawing an individual trajectory) is not applicable to all second-order nonlinear differential equations, while the present method is applicable to every secondorder autonomous system described by the differential equation $\ddot{x}=F(x, \dot{x})$.

\section{Conclusions}

A new, simple, general, rapid, and useful method is presented to determine the behavior of nonlinear second-order autonomous systems for all pertinent initial conditions, and for changes in system characteristics.

\section{ACKNOWLEDGMENTS}

The authors are grateful to Prof. H. N. Ramachandra Rao, Prof. S. Ramaseshan, Dr. C. Ramasastry, and Prof. P. Venkata Rao for their encouragement and discussions during the preparation of this paper.

\section{REFERENCES}

[1] Gibson, John E.. Nonlinear Automatic Control, New York: McGraw-Hill, 1963,

[2] West, John C., Analytical Techniques for Nonlinear Control Systems, London:

31 Thglish Lniversity Press, 1960, ch 4 . ${ }^{2}$ alysis and Design of Nonlinear Feedback Control Systems, New York: McGraw-Hill, 1962.

[4] Ku, Y. H., Analysis and Control of Nonlinear Systems, New York: Ronald, 1958 , chs 1 and 4 .

[5] Szegö, G. P. A new procedure for plotting phase-plane trajectories, AIEE Trans., pt 2, Jul 1962, pp 120-125.

(6) Davis. H. T.. Introduction to Nonlinear Differenticl and Integral Equations, 1.. S. A tomic Energy Commission, 1960, pp 25-32.

DEE Tran, B. IEEE Trans, on Applications and Industry, Jul 1964. pp 258-262.

\section{An Analytical Method for Obtaining the Root Locus with Positive and Negative Gain}

\section{CHI S. CHANG, MEMBER, IEEE}

Abstract-An analytical procedure for obtaining the root locus with positive and negative gain is described. Solutions of various systems consisting of rational polynomial and/or transcendental functions of $s$ are obtained. The root locus for a varying time constant is investigated. Accurate root locus curves can be plotted from the analytical solution. The gain or time constant is calculated for each point on the root locus. Application of the method is particularly suitable for obtaining the root locus of a characteristic equation containing a transcendental function or with a varying time constant for which the graphical method is difficult to apply.

\section{INTRODLCTION}

The root locus is defined to be the trajectory in the $s$ plane, followed by a root of the characteristic equation as some parameter of the corresponding system is varied continuously. The characteristic equation of any system can be written in the form

$$
1+G(s) H(s)=0
$$

The function $G(s) H(s)$ in general may be a rational polynomial and /or a transcendental function of $s$. The graphical method [1], [2], [3] based on the angle condition

$$
\underline{/ G(s) H(s)}=\pi+2 n \pi, \quad n=0, \pm 1, \cdots
$$

has been well developed, but it is a trial-and-error procedure. It can therefore be tedious and inaccurate. In such cases, an analytical solution is preferable. From the analytical solution, an accurate calibrated root locus can be obtained. The analytical procedure for obtaining the root locus of (1) is described in this paper. Analytical solutions of the root locus for some practical systems are obtained and discussed, The gain or time constant is computed analytically. The application of this method to solve higher-order algebraic equations is discussed.

\section{Axalytical Solutions}

The function $G(s) H(s)$ in (1) can be written in the form

$$
G(s) H(s)=\frac{K N(s)}{D(s)}
$$

where $K$ is a parameter which is proportional to the sy'stem gain. Substituting (2) into (1) yields

$$
D(s)+K N(s)=0
$$

Let $s=x+j y$. From the binomial theorem, it can be shown that

$$
s^{n}=\sum_{k=0}^{n / 2} \frac{(-1)^{k} n !}{(2 k) !(n-2 k) !} x^{n-2 k} y^{2 k}+j y \sum_{k=0}^{(n-1) / 2} \frac{(-1)^{k} n ! x^{n-2 k-1} y^{2 k}}{(2 k+1) !(n-2 k-1) !}
$$

where $x$ and $y^{\prime}$ are real variables. Then, (3) implies the two conditions

$$
\begin{aligned}
& R_{e} D(x+j y)+K R_{e} N(x+j y)=0 \\
& I_{m} D(x+j y)+K I_{m} N(x+j y)=0
\end{aligned}
$$

where $R_{\mathrm{e}}$ indicates the real part, and $I_{m}$ indicates the imaginary part of a complex function. The gain constant obtained from (5) is given by

$$
K=-\frac{R_{e} D(x+j y)}{R_{e} N(x+j y)}
$$

Inserting this value of $K$ into (6), the equation of the root locus can be put in the form

$$
y\left[R_{e} N(x+j y) \frac{I_{m} D(x+j y)}{y}-R_{\varepsilon} D(x+j y) \frac{I_{m} N(x+j y)}{y}\right]=0
$$

Reference to (4) shows that the $I_{m} s^{n}$ has a factor $y$. Therefore ( 8 ) also has a factor $y$, revealing that the real axis, $y=0$, is a part of the root locus, and the corresponding gain is $K=-D(x) / N(x)$, which may be either positive or negative. If $D(s)$ and $N(s)$ are polynomial in $s$, then in view of (4), the factor inside the bracket of $(8)$ is a function of $y^{2}$ and $x$. For many practical control systems, the equation of root locus can be solved for $y^{2}$ in terms of $x$; then the root locus can be obtained with ease.

\section{EXAMPLES}

1) If

$$
G(s) H(s)=\frac{K}{\left(s+\sigma_{1}+j \omega_{1}\right)\left(s+\sigma_{1}-j \omega_{1}\right)\left(s+\sigma_{2}\right)} .
$$

where $\sigma_{1}, \omega_{1}, \sigma_{2}$ are real numbers. The solutions are

$$
\left\{\begin{array}{l}
y=0 \\
K=-\left[\left(x+\sigma_{1}\right)^{2}+\omega_{1}^{2}\right]\left(x+\sigma_{2}\right)
\end{array}\right.
$$

and

$$
\left\{\begin{array}{l}
y^{2}=3 x^{2}+\left(4 \sigma_{1}+2 \sigma_{2}\right) x+\sigma_{1}{ }^{2}+2 \sigma_{1} \sigma_{2}+\omega_{1}^{2} \\
K=y^{2}\left(3 x+2 \sigma_{1}+\sigma_{2}\right)-\left[\left(x+\sigma_{1}\right)^{2}+\omega_{1}^{2}\right]\left(x+\sigma_{2}\right)
\end{array}\right.
$$

The second set of equations represents the root locus in the complex portion of the $s$ plane. Using the condition $y=0$ permits evaluation of $x$ at the break-in and break-away points. The result is

$$
x=\frac{-\left(2 \sigma_{1}+\sigma_{2}\right) \pm \sqrt{\left(\sigma_{1}-\sigma_{2}\right)^{2}-3 \omega_{1}^{2}}}{3}
$$

Case I. If $\left(\sigma_{1}-\sigma_{1}\right)^{2}>3 \omega_{1}^{2}$, there are break-in and break-away points. The root loci are the real axis plus a pair of hyperbola as shown in Fig. 1(a). 